\title{
Enhanced Relaxation and Intermixing in Ge Islands Grown on Pit-Patterned Si(001) Substrates
}

\author{
T. U. Schülli, ${ }^{1, *}$ G. Vastola, ${ }^{3}$ M.-I. Richard, ${ }^{1,2}$ A. Malachias, ${ }^{2}$ G. Renaud, ${ }^{1}$ F. Uhlík, ${ }^{3}$ F. Montalenti, ${ }^{3}$ G. Chen, ${ }^{4}$ L. Miglio, ${ }^{3}$ \\ F. Schäffler, ${ }^{4}$ and G. Bauer ${ }^{4}$ \\ ${ }^{1}$ CEA Grenoble, INAC/SP2M, 17 rue des martyrs, F-38054 Grenoble, France \\ ${ }^{2}$ ESRF, Grenoble 6 rue J. Horowitz, F-38043 Grenoble, France \\ ${ }^{3}$ L-NESS and Materials Science Department, University of Milano-Bicocca, Via R. Cozzi 53, I-20125 Milano, Italy \\ ${ }^{4}$ Johannes Kepler Universität Linz, A-4040 Linz, Austria \\ (Received 24 October 2008; published 16 January 2009)
}

\begin{abstract}
We compare elastic relaxation and $\mathrm{Si}-\mathrm{Ge}$ distribution in epitaxial islands grown on both pit-patterned and flat $\mathrm{Si}(001)$ substrates. Anomalous x-ray diffraction yields that nucleation in the pits provides a higher relaxation. Using an innovative, model-free fitting procedure based on self-consistent solutions of the elastic problem, we provide compositional and elastic-energy maps. Islands grown on flat substrates exhibit stronger composition gradients and do not show a monotonic decrease of elastic energy with height. Both phenomena are explained using both thermodynamic and kinetic arguments.
\end{abstract}

DOI: 10.1103/PhysRevLett.102.025502

Ordering, shape and size uniformity of epitaxial Ge (or $\mathrm{SiGe})$ islands on $\mathrm{Si}(001)$ is very important whenever microelectronic applications based on such nanostructures are concerned [1]. Island formation is known to follow the Stranski-Krastanow (SK) growth mode [2], allowing for partial elastic energy relaxation. Significant intermixing between $\mathrm{Si}$ and $\mathrm{Ge}$ within the islands has been reported [3-8], and the role of alloying for growth has been theoretically investigated [9-13]. Since on flat substrates islands tend to nucleate randomly, substrate patterning can be used to achieve controlled positioning [14]. Remarkably, patterning was also shown to increase size uniformity, possibly because of a more regular distribution of capture areas [15]. The growth of ordered nanometric islands with a narrow distribution in shape and size is already exciting per se, but recent results $[15,16]$ indicate that the influence of patterning can be even more far reaching. Self organized patterning in ultra high vacuum (UHV) may also be used in the future, in order to control size and relaxation in SiGe islands [17]. We recall that $\mathrm{SiGe}$ islands on $\mathrm{Si}$ are coherent up to a critical volume $V_{c}$, characteristic for the onset of the formation of misfit dislocations [18]. In Ref. [15], it was demonstrated that patterning of $\mathrm{Si}(001)$ extends the allowed volume range for coherent islands. According to atomistic and finite element method (FEM) calculations reported in the same paper, this can be explained by an extra relaxation, caused by the pit, which lowers the substrate or island misfit. The possibility of controlling also the relaxation level of the islands by growth on patterned substrates appears extremely intriguing. In this Letter, we report an experimental proof of the abovementioned effect, and we show that the pits influence both, average relaxation and distribution of Ge within the islands.

Pit-patterned substrates were prepared by lithography, following the procedure used in [19]. Seven ML of Ge were deposited at $650 \hat{\mathrm{A}}{ }^{\circ} \mathrm{C}$ on a $\mathrm{Si}(001)$ substrate with a $900 \times$
PACS numbers: $81.16 . \mathrm{Nd}, 61.46 .-\mathrm{w}, 71.15 . \mathrm{Pd}, 81.07 .-\mathrm{b}$

$900 \mu \mathrm{m}^{2}$ part of it being patterned with pits at a periodic spacing of $495 \mathrm{~nm}$. The growth of a Si buffer prior to Ge deposition leaves periodic downward pyramid-shaped $\{1110\}$ pits [19]. The sample was transferred under $\mathrm{N}_{2}$ atmosphere into the UHV chamber of beam line BM32 at the ESRF in Grenoble. AFM analysis shows that Ge islands nucleate at the bottom of these pits and form dome-shaped islands with identical facets as on the flat part of the substrate. The average island density per surface unit area is $5 \mu \mathrm{m}^{-2}$ for the patterned part and $20 \mu \mathrm{m}^{-2}$ for the flat one. The domes on the patterned (flat) part have an average height of $28 \mathrm{~nm}(23 \mathrm{~nm})$ and a diameter of $130 \mathrm{~nm}$ $(110 \mathrm{~nm})$.

The islands were characterized by grazing incidence $x$ ray diffraction (GIXD) at an x-ray energy of $11.04 \mathrm{keV}$. The incident beam and the collimation on the detection side were translated in order to select between patterned and flat regions. Reciprocal space maps in the vicinity of the (400) Bragg reflection were recorded, as shown in Figs. 1(a) and 1(b) together with corresponding AFM images (insets). The intensity distribution along the radial direction clearly extends to higher lattice parameters for the patterned region. The islands grown on the flat have their main lattice parameter around $5.465 \AA$ (in reciprocal lattice units of $H=3.975$ ) whereas for the patterned part, the lattice parameters are stretched in reciprocal space, showing a maximum at $\sim 5.51 \AA(H=3.943)$.

To understand the observed differences, we related the lattice-parameter to the local Ge content inside the islands by exploiting anomalous $\mathrm{x}$-ray scattering. By monitoring the scattered intensity when varying the x-ray energy in the vicinity of the Ge $K$ edge $\left(E_{e}=11.103 \mathrm{keV}\right)$ at a fixed momentum tranfer $Q$, the corresponding average Ge concentration can be determined, so that in-plane lattice parameter $a_{\|}$vs Ge content $\left(c_{\mathrm{Ge}}\right)$ data are extracted without any model assumption $[5,20]$. It is found that for the islands 


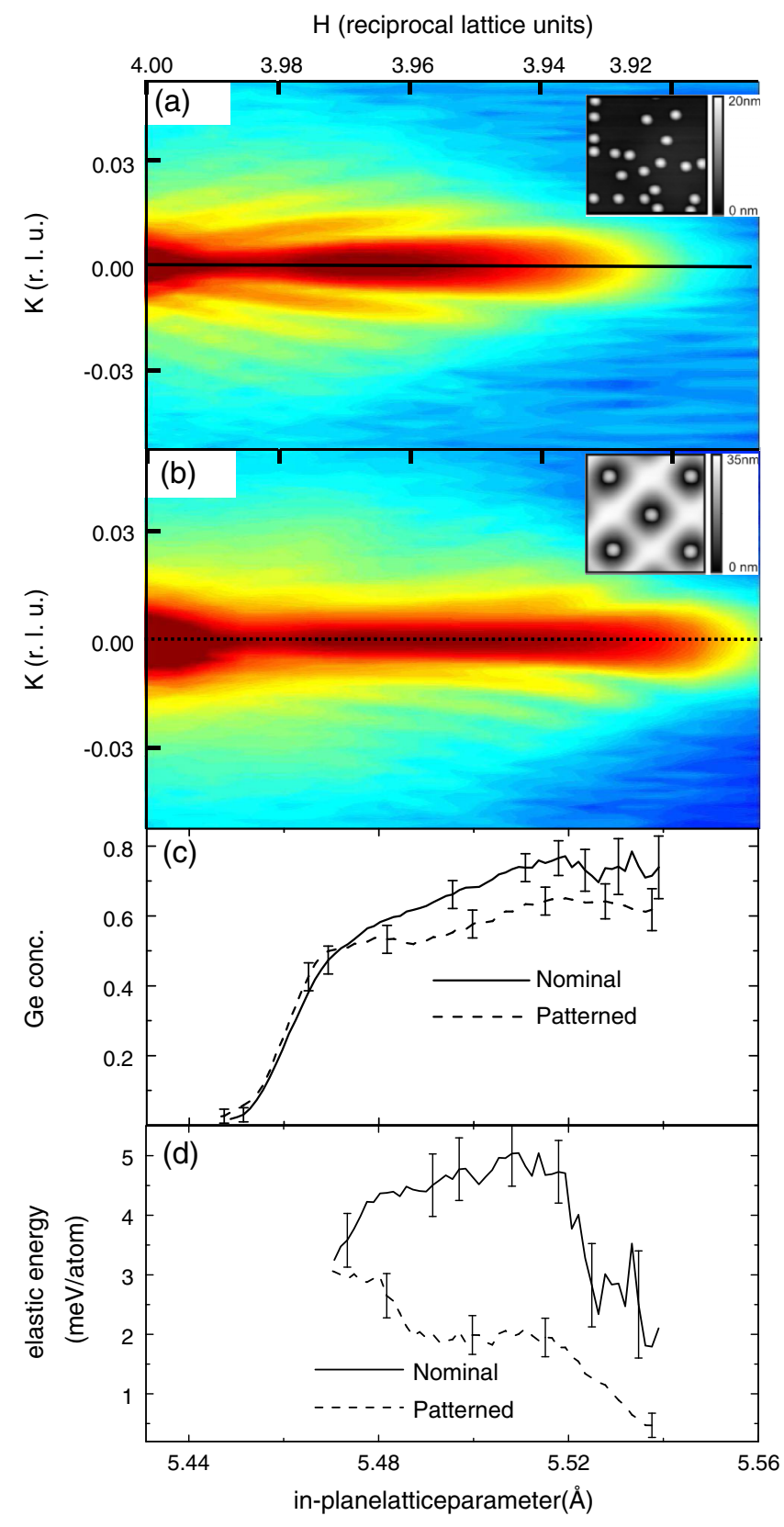

FIG. 1 (color online). Logarithmic intensity distribution in the vicinity of the (400) Bragg reflection for Ge islands grown on the flat (a) and patterned (b) sample part. Corresponding AFM images $\left(1 \mu \mathrm{m}^{2}\right.$ in size) are shown as insets. (c) Ge content inside the islands as a function of lattice parameter for growth on the flat (full line) and on the patterned part (dashed line). (d) elastic energy as a function of lattice parameter as extracted from the x-ray data. All figures correspond to the same latticeparameter scale.

grown on the flat, the maximum Ge concentration for highest lattice parameters is slightly higher than for the patterned case [Fig. 1(c)]. However, as visible from the intensity distribution in Figs. 1(a) and 1(b), these relaxed regions contribute little to the total diffracted intensity. The combined analysis of the x-ray size oscillations, and the island shape as resolved by AFM, yield that the major part of the island volume is situated at lattice parameters below $5.48 \AA$ for islands on the flat. Combining x-ray diffraction and AFM, the mean Ge content is determined to be $c_{\mathrm{Ge}} \simeq$ $(60 \pm 5) \%$ for both island types. Hence, the presence of the pits does neither influence the shape nor the mean $\mathrm{SiGe}$ composition of the islands. Let us see where, instead, differences exist. Figure 1(c) shows $c_{\mathrm{Ge}}$ vs $a_{\|}$for the flat (full lines) and the patterned region (dashed lines). The continuous broadening of $\mathrm{H}$-constant profiles for lower $\mathrm{H}$ values points to a monotonic lattice parameter increase as a function of height in the islands [21]. The flatness of the dashed curve indicates lower gradients for $c_{\mathrm{Ge}}$ inside the islands grown in pits in comparison to the flat case. It is thus clear that the elastic energy at comparable $c_{\mathrm{Ge}}$ must be significantly lower for islands nucleating in the pits, as the intensity in reciprocal space stretches out to much higher lattice parameters. The volumetric elastic energy can be extracted from these data, as performed in Refs. [7,20]. It is plotted as a function of lattice parameter in Fig. 1(d). In the narrow region that forms the interface between island and substrate, and hence the region where the in-plane strain $\epsilon_{\|}$ changes sign from expansive to compressive nature, our method does not allow for a precise determination of the elastic energy since regions with similar lattice parameter and slightly different composition will have a mean $\bar{\epsilon}_{\|}$that amounts to zero. However, with the elastic energy $E_{e} \propto \epsilon_{\|}^{2}$, its mean value does not average to zero. In the region of validity, our method yields a remarkable decrease of elastic energy throughout the islands grown on the pit-patterned part. One has to state that such x-ray measurements carry information on the in-plane lattice parameter, but effects of local hydrostatic compression cannot be taken into account when determining the elastic energy directly from the data.

More insights can be gained by extracting the actual 3D Ge distribution and the elastic-energy profile throughout the island. Over the last ten years, $\mathrm{x}$-ray methods have been developed, coupling lattice parameter with lateral size [21] and chemical composition of an object, to reconstruct a concentration profile inside nanostructures $[4,5,22]$. These methods are limited, however, by model assumptions concerning the shape of the considered isostrain regions. A combination of $\mathrm{x}$-ray methods and FEM calculations is thus necessary in order to overcome the lack of experimentally accessible information. We have used a fitting procedure that exploits a FEM-based treatment of nonuniform concentration profiles, to determine the local $\mathrm{Ge}$ content without any constraint. After the island and substrate geometry is created based on AFM images, nonuniform concentration values are assigned on a discrete mesh [13]. The elastic problem is then solved exploiting linear interpolation, $\left(c_{\mathrm{Ge}}, a_{\|}\right)$data are extracted, and the statistical $\chi^{2}$ is evaluated by comparison with the corresponding $\mathrm{x}$-ray data. Using an iterative procedure based on local concentration exchanges, $\chi^{2}$ is minimized. Technical details on the way we handle nonuniform distributions within FEM can be found in [13] where the elastic energy was 
minimized. In the present work, the deviation from the experimental data is minimized. It is important to emphasize that particular care is required in assigning the correct statistical weight to the data. During the fit, $\left(c_{\mathrm{Ge}}, a_{\|}\right)$pairs from the FEM grid were computed from the island interior and from a surrounding region in the substrate with a lateral extension inferred from the experimental island density, and a depth of $12 \mathrm{~nm}$, i.e., the estimated penetration depth of the x-rays in our setup. This allowed us to filter out the substrate contribution from the experimental data which, as shown in Fig. 1(c) for the flat case, causes a sudden drop of the average Ge content at low lattice parameters, due to the strong scattering contribution from the Si-substrate. From the x-ray data, it is thus difficult to analyze the region of the substrate-island interface. Another difficulty stems from the vanishing experimental scattering volume of the regions with lattice parameters close to bulk Ge. In the fit shown in Fig. 2, the local value of $c_{\mathrm{Ge}}$ was extrapolated from experiments, assigning a large error bar in order not to bias the region where real data were present. Finally, a satisfactory fit (requiring $\sim 10^{4}$ iterations) is obtained, the full solution of the elastic problem being required at each step. The FEM fitted data (small dots in Fig. 2) show that several concentration values can be associated with a single value of lattice parameter, which is not obvious from the experimental curve which averages them out.

The 3D compositional maps corresponding to our best fits are displayed in Figs. 3(a)-3(d) together with their horizontally averaged values [Fig. 3(e)], while the elastic energy per atom is reported in Fig. 3(f), where a comparison with the uniform composition case is also shown. In the flat case-Figs. 3(a) and 3(b) - an almost pure Si region is found close to the base edges, a result fully compatible with selective-etching data [23]. From Figs. 3(c) and 3(d), one sees that $\mathrm{Si}$ enrichment at the base is less evident in the pit case where the overall Ge distribution is more uniform, but for more localized irregularities. This is particularly evident from the horizontally averaged $c_{\mathrm{Ge}}$ values dis-

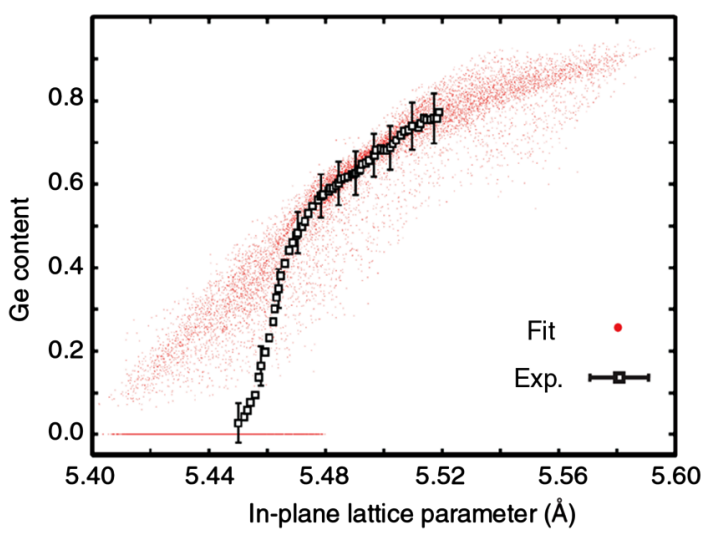

FIG. 2 (color online). Experimental data of the Ge-content as a function of lattice parameter together with FEM fitted data after proper separation of the Si substrate contribution (flat case). played in Fig. 3(e). The different Ge distribution of the island apex along the [100] and [110] cross sections is due to the fact that the island boundary is composed by a set of facets and edges which is different for the two sections. It has thus a direct influence on the local elastic relaxation and hence the Ge concentration profile [24]. The above observations can be justified using both thermodynamic and kinetic arguments. The edges of the islands grown on flat substrates are the most compressed regions in case a uniform Ge distribution is considered [25], so that replacing $\mathrm{Ge}$ with $\mathrm{Si}$ atoms produces significant relaxation. Comparing the elastic energy in flat vs pit-patterned substrates [Fig. 3(f)] in the case of a uniform distribution, one sees that the driving force for Si segregation at the island base is weaker in pits, since the energy difference between base and top is smaller. Entropy of mixing could then prevail over elastic relaxation, forcing enhanced intermixing. While this thermodynamic argument supports our
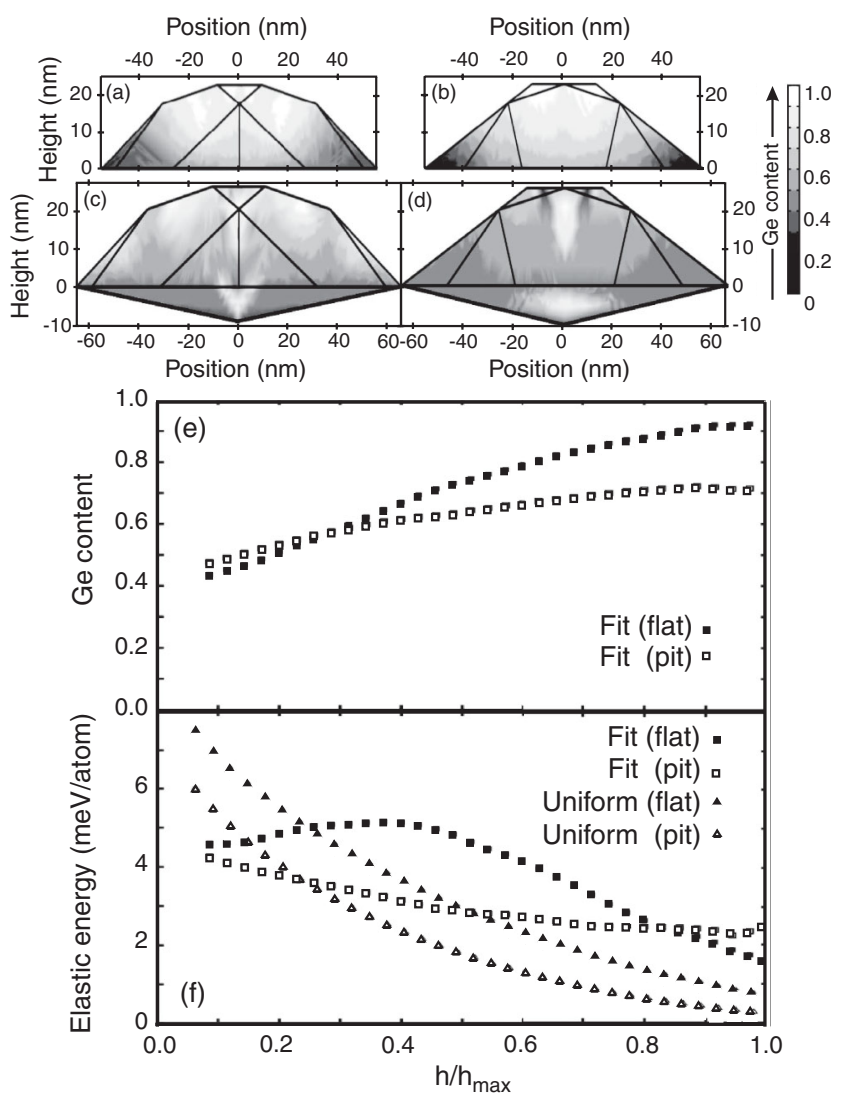

FIG. 3. Cross section map of the 3D-reconstructed Ge content profile for islands grown on flat (a), (b) and patterned (c), (d) samples. Sections are shown along the [100] (a), (c) and the [110] (b), (d) crystallographic directions. Panel (e) displays the average Ge content as a function of height. Panel (f) reports the elastic energy profile corresponding to the fitted Ge distribution on flat (filled box) and patterned (empty box) substrates. Plots made for the case of uniform concentration are analogously displayed for flat (filled triangles) and pit (empty triangles) case. The quantity $h / h_{\max }$ is a normalized height to allow the proper comparison between both island types at every height $h$. 
results, it is important to consider that during growth, kinetic limitations are present, so that one should always check whether the proposed scenario is favored or simply made possible by plausible kinetic paths. On flat substrates, large amounts of Si become available during growth when the strong compressive stress at the edges produces trenches penetrating the $\mathrm{Si}$ substrate [26]. These $\mathrm{Si}$ atoms coming out close to the island periphery could easily be incorporated in the regions which are most favorable from the energetic point of view. As a result, the final profile resembles the minimum-energy one [13]. In the initial stages of growth, trenches are not yet present, providing a kinetic justification for the limited lateral extension of the external Si-rich region. Our results do not confirm the formation of a central Si-rich region $([4,7])$, whose presence seems to be difficult to explain from both the thermodynamic and kinetic point of views. In pits, the Si-supply mechanism could be different. In Ref. [27], data collected on pit-patterned samples similar to ours indicated that the condition of a perfect WL completed prior to island formation is not fulfilled. Since preliminary results on the present samples confirm this observation, we believe that in pits, enhanced Si supply prior to trench formation is possible, i.e., from detachment of uncovered $\mathrm{Si}$ atoms from the steps of the pits' facets. These could reach the growing islands by surface diffusion, leading to a more uniform intermixing profile.

Let us now analyze quantitatively the main differences between the flat and the patterned case in terms of elastic energy. From Fig. 3(f), one sees that the presence of the pit allows for a significant decrease $(\sim 30 \%$ on average) in elastic energy. Since this effect is confirmed also for uniform Ge distributions [triangles in Fig. 3(f)], the key role must be played by the different geometry. Before island formation, the pit is filled by Ge forming an inverted pyramid. This redistributes the load between Ge and the surrounding Si resulting in better strain relaxation with respect to a flat WL so that islands on pits nucleate on a substrate with a lower effective misfit. This relaxation mechanism was demonstrated in [15]. Additionally, the behavior of the elastic energy shows that the usual picture of a base-to-top stress relaxation breaks down in the case of a flat substrate [25]: the curve displayed in Fig. 3(f) clearly displays a maximum. Combining this with Fig. 3(e), one sees that $\mathrm{Si}$ enrichment at the base guarantees a lowering of the elastic energy. As soon as $c_{\mathrm{Ge}}$ approaches its average value (at around $h / h_{\max }=0.3$ ), elastic-energy lowering becomes less efficient: the strong elastic load determined by the high Ge content cannot be relieved by the limited deformation in regions still close to the base. In the proximity of the island top, instead, the elastic energy is nicely lowered in spite of the maximum Ge content in the island.

If prepatterning is already seen as a powerful tool to control positioning and homogeneity of heteroepitaxial islands, we have shown that it also allows one to control elastic-energy release and thus to grow islands with the desired relaxation. The kinetics of Si supply seems to be also influenced by the pit, calling for further studies for achieving full control.

Financial support by the EU-funded project "D-DotFET" and the FWF (Vienna, project SFB025) and GMe (Vienna) are gratefully acknowledged, together with technical help received from R. Gatti.

*schulli@esrf.fr

[1] F. Schäffler, Semicond. Sci. Technol. 12, 1515 (1997).

[2] V. A. Shchukin and D. Bimberg, Rev. Mod. Phys. 71, 1125 (1999).

[3] G. Capellini, M. De Seta, and F. Evangelisti, Appl. Phys. Lett. 78, 303 (2001).

[4] A. Malachias et al. Phys. Rev. Lett. 91, 176101 (2003).

[5] T. U. Schülli et al., Phys. Rev. Lett. 90, 066105 (2003).

[6] G. Medeiros-Ribeiro et al., Appl. Phys. A 80, 1211 (2005).

[7] G. Medeiros-Ribeiro and R. S. Williams, Nano Lett. 7, 223 (2007).

[8] A. Rastelli et al., Nano Lett. 8, 1404 (2008).

[9] C. Lang, D. J. H. Cockayne, and D. Nguyen-Manh, Phys. Rev. B 72, 155328 (2005).

[10] G. Hadjisavvas and P. C. Kelires, Phys. Rev. B 72, 075334 (2005).

[11] Y. Tu and J. Tersoff, Phys. Rev. Lett. 98, 096103 (2007).

[12] N. V. Medhekar, V. Hegadekatte, and V. B. Shenoy, Phys. Rev. Lett. 100, 106104 (2008).

[13] R. Gatti, F. Uhlík, and F. Montalenti, New J. Phys. 10, 083039 (2008).

[14] Z. Zhong and G. Bauer, Appl. Phys. Lett. 84, 1922 (2004).

[15] Z. Zhong et al., Phys. Rev. Lett. 98, 176102 (2007).

[16] H. Hu, H. J. Gao, and F. Liu, Phys. Rev. Lett. 101, 216102 (2008).

[17] G. Katsaros et al., Phys. Rev. Lett. 101, 096103 (2008).

[18] A. Marzegalli et al., Phys. Rev. Lett. 99, 235505 (2007).

[19] G. Chen et al., Phys. Rev. B 74, 035302 (2006).

[20] R. Magalhães-Paniago et al., Phys. Rev. B 66, 245312 (2002).

[21] I. Kegel et al., Phys. Rev. Lett. 85, 1694 (2000).

[22] A. Letoublon et al., Phys. Rev. Lett. 92, 186101 (2004).

[23] G. Katsaros et al., Phys. Rev. B 72, 195320 (2005).

[24] In Figs. 3(c) and 3(d), the calculations show Ge rich regions of the pit bottoms which do not appear in 3(e). In this figure, we make the comparison between the average composition between islands on flat and patterned substrates. Since the material at the bottom of the pit is of course not present in the island on flat substrate, the averaged compositions of Fig. 3(e) do not take into account this volume.

[25] G. Vastola, R. Gatti, A. Marzegalli, F. Montalenti, and Leo Miglio, Detailed Analysis of the Shape-Dependent Deformation Field in $3 D$ Ge Islands on Si(001) (Springer, Berlin, 2008), pp. 421-438.

[26] S. A. Chaparro, Y. Zhang, and J. Drucker, Appl. Phys. Lett. 76, 3534 (2000).

[27] T. Stoica et al., Nanotechnology 18, 455307 (2007). 